\title{
Bleaching treatment in devitalised teeth and the restoration of anterior aesthetics: 2 case reports
}

\begin{abstract}
Discolouraton in anterior teeth is a cosmetic problem that definitely requires treatment in terms of patient psychology. Despite several alternatives such as crowns and laminate veneers in discolouration treatment, the first treatment of these teeth should be with bleaching methods. Whitening procedures are a treatment option that is easier to apply, less expensive and more conservative than other restorative methods. Although the secret of success in these treatments is knowing what caused the discolouration, it is also related to correct diagnosis and the correct decision of which whitening technique to use. The aim of this paper was to present the results of techniques used to restore the aesthetics of patients who presented with complaints of discolouration of the anterior teeth.
\end{abstract}

Volume 8 Issue 6 - 2017

\section{Suzan Cangul, Salih Aydin}

Department of Restorative Dentistry, Dicle University, Turkey

Correspondence: Suzan Cangul, Department of Restorative Dentistry, Dicle University, 21280, Diyarbakir,Turkey,

Emailsuzanbali@outlook.com

Received: October 28, 2017 | Published: November 08, 2017

Keywords: bleaching, composite, discolouration

\section{Introduction}

Discolouration of devitalised teeth, especially in the anterior region leads to significant aesthetic problems. This discolouration which is generally seen in a single tooth can also involve several teeth with the effect of trauma. The reasons for this can be remaining pulp tissue after root canal treatent, resorption of roots, haemorrhage within the pulp, necrotic pulp and materials used during endodontic and restorative treatment. ${ }^{1}$ In the treatment of discolouration, which has negative psychological effects on the patient, agents such as hydrogen peroxide, carbamide peroxide and sodium perborate are used. ${ }^{2}$ Whitening agents are applied in various ways including thermocatalytic, walking bleach, home-type or office-type whitening and combined techniques. Of these techniques, the currently most widely used is the walking bleach technique, which has the advantages of being simple, rapid and safe to apply. ${ }^{3,4}$ In the walking bleach technique, sodium perborate and $30 \%-35 \%$ hydrogen peroxide are mixed and placed in the entrance to the cavity. This is then temporarily closed with glass ionomer cement or composite resin. Until the whitening occurs, the patient is called for examination at 2-3-day intervals. At each session, the same procedure is repeated. After completion of the bleaching procedure, the tooth is restored with composite material. The most important point requiring attention in bleaching treatment is that the canal treatment has been well made to the tooth to which the devital bleaching is to be applied. This treatment must be completed in such a way as to prevent the apical entry of micro-organisms or other substances. ${ }^{5}$ Following bleaching treatment the permeability of the enamel may be increased. In addition, bleaching treatments may cause external cervical resorption and agents such as hydrogen peroxide lead to apical leakage of defects in the cement. ${ }^{6}$ The aim of this paper was to present the successful aesthetic results of treatment to eliminate the problems of two patients who presented with complaints of discolouration.

\section{Case I}

A 23-year old male presented at the Restorative Dental Treatment Department of the Dentistry Faculty of Dicle University with the complaint of discolouration in the right upper central tooth and diastema between the central teeth. From the patient anamnesis it was learned that the patient had a history of trauma from 7 years previously. As a result of the clincal and radiological examinations, it was seen that canal treatment had been applied to the right upper central tooth and there was a loss of substance. Before the treatment, intraoral photographs were taken of the patient and it was aimed to provide anesthesia of upper anterior teeth at the request of the patient (Figure 1,2). First, gingivectomy was applied to the upper right central tooth with the aim of levelling the crown lengths of the central teeth. Then it was decided to apply devital bleaching to the same tooth. After making an opening to the cavity, the root canal filling was cleaned to $2 \mathrm{~mm}$ below the enamel cement border. The dentin remnants were cleaned using sodium hypochloride followed by EDTA solutions. The floor of the cavity was then closed with glass ionomer cement. As the bleaching agent, $37 \%$ hydrogen peroxide was used and this was applied to the whole pulp chamber. After placing a fine cotton gauze over the bleaching agent, the cavity was temporarily closed with zinc oxide eugenol. The same procedures were repeated in 3 sessions at 2-day intervals. When sufficient bleaching was observed, the cavity was thoroughly washed out with distilled water and dried then a pad of calcium hydroxide $\left(\mathrm{Ca}(\mathrm{OH})_{2}\right)$ was placed to neutralise the hydrogen peroxide activity. Cotton gauze was placed over the pad and the cavity was closed with temporary filling material (Figure 3). In the final stage the $\left(\mathrm{Ca}(\mathrm{OH})_{2}\right)$ pad was washed and dried, then the cavity was closed with cavit. The length of the tooth crown was extended while performing the gingivectomy operation in the bleaching process. Immediately after this operation and completion of the bleaching treatment, a fibre post was applied (Figure 4). This was placed after cleaning the canal filling material in both the root ends. After evaluation with periapical films, full restoration of the tooth was made with composite resin (Figure 5). Then the procedure was applied to close the diastema between the upper central teeth. The teeth were completely isolated with cotton wool rolls then acid was applied for 15 secs to the surfaces to be restored. After washing and drying the surfaces applied with acid, adhesive resin was applied to these surfaces and polymerisation was made with an LED light source for 10 secs. Then, with the aid of a clear band, composite resin was 
applied with incremental technique and the diastema was closed. Any excess was removed with microgranulated flame tipped burrs and the finishing and polishing procedures were made with discs and rubbers (Figure 6,7). The patient was recalled for control after 6 months. No problems were observed in the teeth.

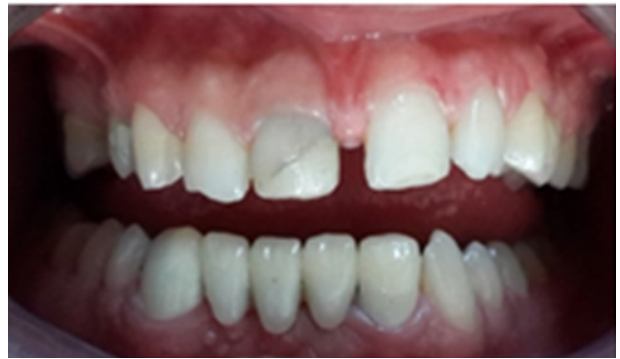

Figure I Photograph of the first image of the patient's upper central teeth.

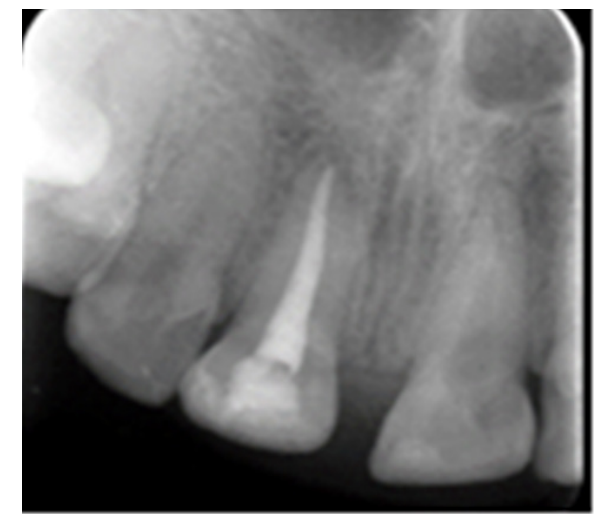

Figure 2 Radiographic image of upper right central tooth.

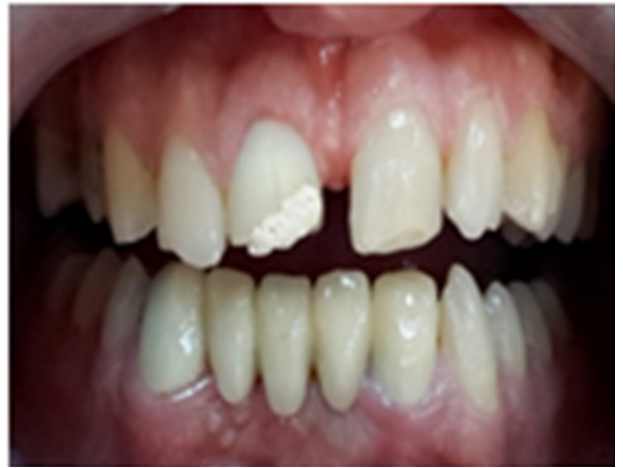

Figure 3 Post-bleaching and gingivectomy image of upper right central tooth.

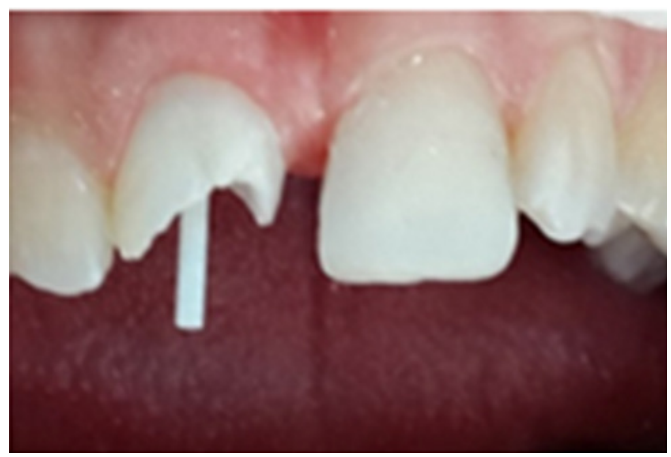

Figure 4 Fiber post application in upper right central.

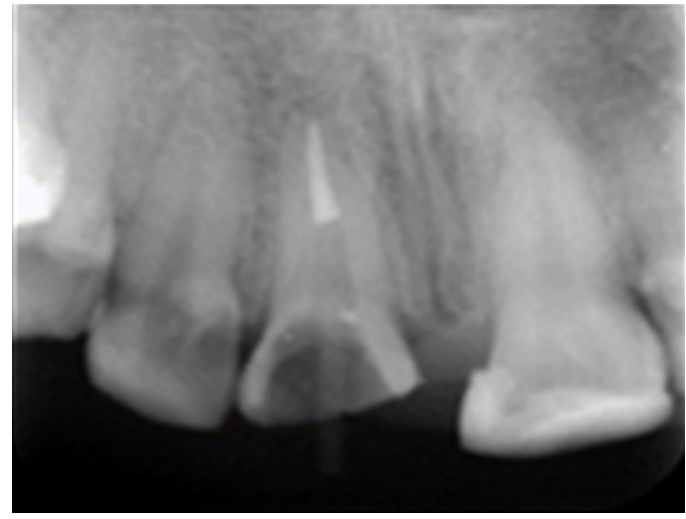

Figure 5 Radiographic image of upper right central tooth with fiber post placed.
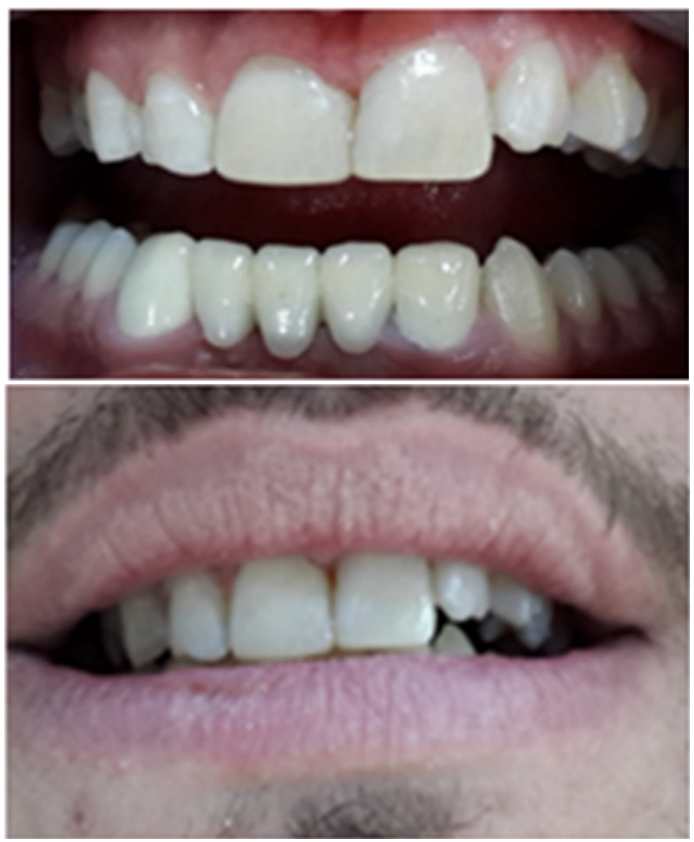

Figure 6 \& 7 Image of anterior teeth after restoration.

\section{Case 2}

A 22-year old female who had suffered an accident in childhood presented at the clinic with aesthetic complaints of the upper anterior teeth. In the clinical examination, there was seen to be severe substance loss on the buccal surfaces of the upper right and left central teeth and there was brown discolouration. As there was discolouration in the collar section of the upper left central tooth and intercoronally, a vitality test was applied to the tooth, which showed that that the upper left central tooth is devital, right central tooth is vital. In the radiological examination, no pathology was determined in either of the two teeth (Figure 8,9 ). After all the examinations, intraoral photographs were taken and the first treatment was started of canal treatment to the upper left central tooth (Figure 10). Entrance to the cavity was opened from the buccal surface. After the endodontic treatment, the tooth was temporarily closed with zinc oxide eugenol. The patient was recalled one week later for bleaching treatment. The temporary filling was removed and the entry to the cavity was extended to $2 \mathrm{~mm}$ below the collar level. Then closure was made with 
zinc phosphate over gutta percha (Figure 11). As the bleaching agent, $37 \%$ carbamide peroxide was used and was applied to the whole cavity, which was then covered with a fine sterile gauze. Temporary restoration was made with composite resin. The patient was recalled after 3 days and the tooth bleaching was evaluated. As sufficient bleaching had not been obtained, the cavity was cleaned and the same procedures were repeated. The patient returned after a further 3 days and the tooth colour was observed to have sufficiently lightened. The cavity was opened, washed and dried, then powdered calcium hydroxide was applied to the cavity with an amalgam gun to eliminate the harmful effects of the whitening agent. A small piece of cotton gauze was then applied and temporary closure was made with cavit (Figure 12). One day later, a lamina shape cut was made to the upper right and left central teeth and permanent restoration was made with composite resin (Figure 13,14).

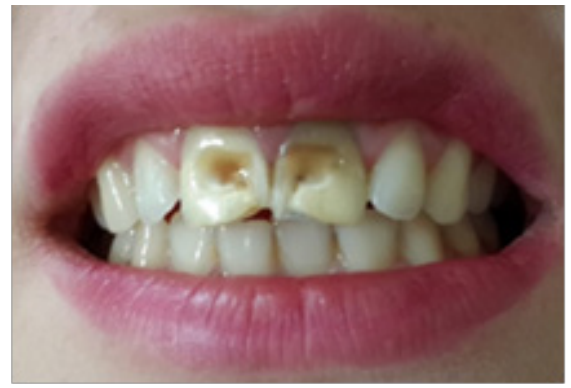

Figure 8 First image of upper central teeth.

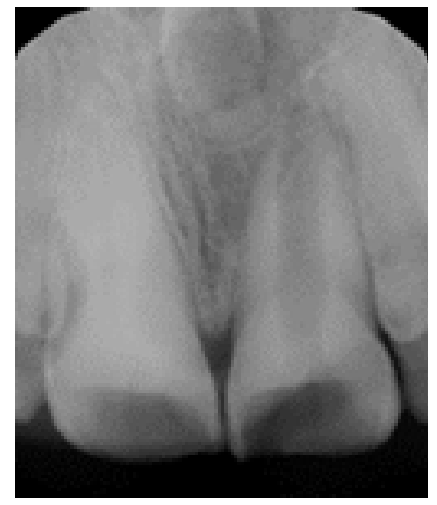

Figure 9 Radiographic image of upper central teeth.

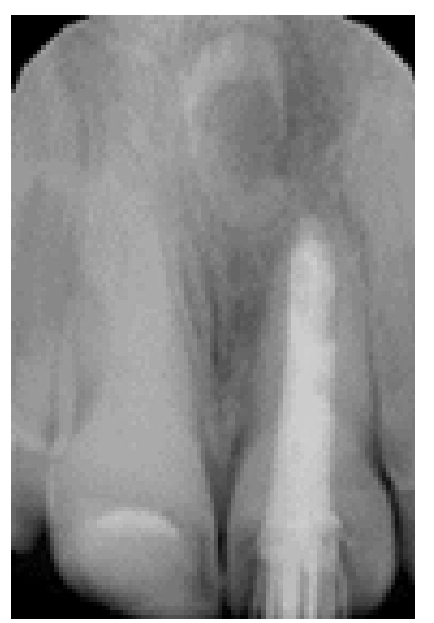

Figure I 0 Radiographic image of the upper left central tooth after endodontic treatment.

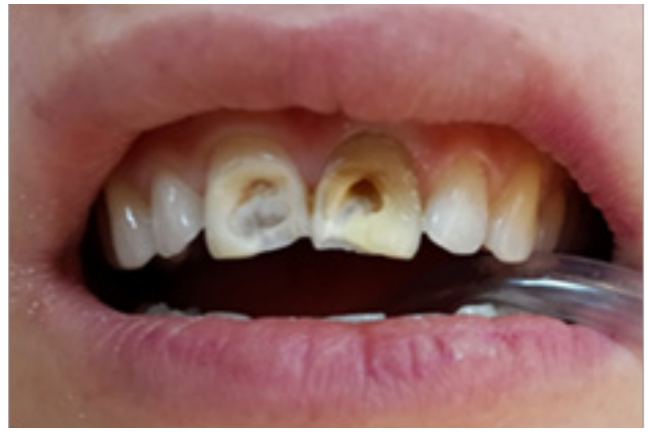

Figure I I The image of the upper central teeth before bleaching treatment.

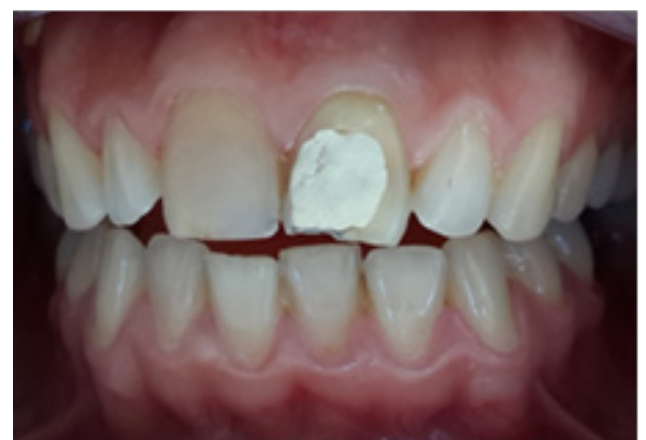

Figure I 2 Image of upper right central tooth after bleaching treatment.
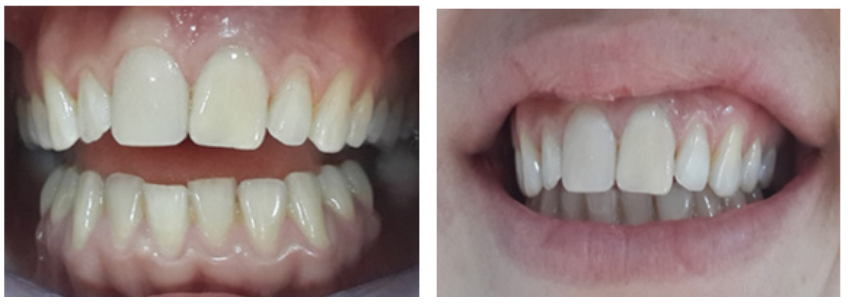

Figure I 3 \& I 4 Image of upper anterior central teeth after composite resin restoration.

\section{Discussion}

The formation of aesthetic defects in the anterior teeth is a situation which absolutely requires treatment. Discoloured teeth in particular affect the psychology of the patient negatively. Therefore, although invasive methods can be used, the application of bleaching treatments is recommended first. ${ }^{7}$ Many factors can cause discolouration of the teeth, but the leading cause is pulp necrosis. In pulp necrosis which develops as a result of bacterial, chemical and mechanical factors, tissue destruction occurs. The tissue remnants emerging as a consequence of this penetrate the dentin tubules and over time cause an increased change of colour in the dentin. In addition to pulp necrosis, several other factors may cause discolouration, such as intrapulpal haemorrhage, developmental defects, systemic diseases, dentin hypercalcification, drugs, age, environmental factors and trauma. In patients with traumarelated discolouration, the bleaching treatment depends on the degree and amount of discolouration. However, the effect is not permanent and there is a possibility of recurrence. These problems are usually patient-related. ${ }^{8-10}$ Other reasons for discolouration can be related to failure during endodontic and restorative treatments. During root canal treatment, insufficient cleaning of the pulp chamber and leaving some of the canal filling material there can cause discolouration, which is related to the dental practitioner. As amalgam restorations 
have metallic components, the colour of the dentin may become grey, and in composite restorations, chemicals entering from the region as a result of leakage stain the dentin. In addition, the tooth may show a dark colour reflecting the metal posts under the composite. The most commonly used bleaching materials are primarily hydrogen peroxide, carbamide peroxide and sodium perborate. Of these agents with an oxidising effect, hydrogen peroxide and carbamide peroxide are generally used at a concentraton of $35 \% .{ }^{11}$ When the root canal filling is not sufficient, there is the possibility of the bleaching agents reaching the apical region. Therefore, researchers such as HansenBayless \& Davis ${ }^{12}$ have recommended the technique of closing the canal opening with materials such as glass ionomer cements, zinc oxide eugenol, intermediate restorative material (IRM), zinc phosphate cements, hydrophyllic materials and composite resins. A study made using glass ionomer cement supported this theory. The devital bleaching technique is not recommended in cases where the discolouration of the enamel is superficial, when there is decay, when there is excessive loss of dentin tissue or when the colour of composite restorations has changed. When various studies are examined it can be seen that devital bleaching can cause several complications. The most frequently encountered is that hydrogen peroxide is effective in cervical root resorption. In a study by Holmstrup et al. ${ }^{13} 69$ of teeth, no resorption was observed at the end of a 3-year follow-up period, whereas Friedmann et al. ${ }^{14}$ reported findings of resorption in $12(6.9 \%)$ of 58 teeth. Furthermore, a 30\% concentration of hydrogen peroxide can cause burns to the gums. To prevent chemical burns such as these, the soft tissues should be covered with an isolation cream. Following bleaching treatment, if these oxidising agents are not completely removed from the cavity, there will be a negative effect on the bonding of the restorative materials to the tooth. Therefore, bleaching agents must be completely cleaned from the cavity before the restoration. ${ }^{15}$ Previous studies have shown that the use of calcium hydroxide to neutralise the effect of whitening agents has no effect on the bonding of composite resins used for the permanent restoration. ${ }^{16}$

\section{Conclusion}

Discolouration in devitalised teeth is one of the leading problems affecting patients in an aesthetic perspective. Treatments to be able to overcome this problem are now being frequently applied. It is important in these treatments that an aesthetically pleasing appearance is provided without disrupting the natural tooth structure. Patients must be fully informed about these treatments and possible complications and they must be called for regular follow-up.

\section{Funding}

None.

\section{Acknowledgments}

None.

\section{Conflicts of interest}

There is no conflicting Interest.

\section{References}

1. Plotino G, Buono L, Grande NM, et al. Nonvital tooth bleaching: a review of the literature and clinical procedures. J Endod. 2008; 34(4):394-407.

2. Perrine G, Reichl R, Baisden M, et al. Comparison of $10 \%$ carbamide peroxide and sodium perborate for intracoronal bleaching. Gen Dent. 1999;48(3):264-270.

3. MacIsaac AM, Hoen C. Intracoronal bleaching: concerns and considerations. J Can Dent Assoc. 1994;60(1):57-64.

4. Hara AT, Pimenta LAF. Nonvital tooth bleaching: A 2-year case report. Quintessence Int. 1999;30(11):748-754.

5. Smith JJ, Cunningham CJ, Montgomery S. Cervical canal leakage after internal bleaching procedures. J Endod. 1992;18(10):476-481.

6. Al-Nazhan S. External root resorption after bleaching: A case report Oral Surg Oral Med Oral Pathol. 1991;72(5):607-609.

7. Soares CJ, Fonseca RB, Martins LRM, et al. Esthetic Rehabilitation of anterior teeth affected by enamel hypoplasia: A case reports. $J$ Esthet Restor Dent. 2002;14(6):340-348.

8. Bizhang $\mathrm{M}$, Heiden $\mathrm{A}$, Blunck $\mathrm{U}$, et al. Intracoronal bleaching of discolored non-vital teeth. Oper Dent. 2003;28(4):334-340.

9. Watts A, Addy M. Tooth discolouration and staining: a review of the literature. Br Dent J. 2001;190(6):309-316.

10. Rodrigues JA, Marchi GM, Ambrosano GM, et al. Microhardness evaluation of in situ vital bleaching on human dental enamel using a novel study design. Dent Mater. 2005;21(11):1059-1067.

11. Rotstein I, Lehr Z, Gedalia I. Effect of bleaching agentson inorganic components of human dentin and cementum. J Endod. 1992; 18(6):290 293.

12. Hansen-Bayless J, Davis R. Sealing ability of two intermediate restorative materials in bleached teeth. Am J Dent. 1992;5(3):151-154.

13. Holmstrup G, Palm AM, Lambjerg-Hansen H. Bleaching of discolored root-filled teeth. Endod Dent Traumatol. 1998;4(5):197-201.

14. Friedman S, Rotstein I, Libfeld $\mathrm{H}$, et al. Incidence of external root resorption and esthetic results in 58 bleached pulpless teeth. Endod Dent Traumatol. 1998;4(1):23-26.

15. Dishman MV, Covey DA, Baughan LW. The effects of peroxide bleaching on composite to enamel bond strength. Dent Mater. 1994;10(1):33-36.

16. Demarco F, Freitas J, Silva M, et al. Microleakage in endodontically treated teeth: influence of calcium hydroxide dressing following bleaching. Int Endod J. 2001;34(7):495-500. 